\title{
Intravitreal Injection of Bevacizumab to Treat a Macular Edema Caused by Leber's Miliary Aneurysm
}

\author{
Masayuki Takeyama Masayoshi Iwaki Masahiro Zako \\ Department of Ophthalmology, Aichi Medical University, Nagakute, Japan
}

\section{Key Words}

Bevacizumab · Intravitreal injection · Leber's miliary aneurysm · Macular edema

\begin{abstract}
Purpose: Leber's miliary aneurysm is a variant of Coats' disease and similar to type 1 idiopathic macular telangiectasia. A recent report showed that an intravitreal injection of bevacizumab (IVB) was effective in an adult patient with type 1 idiopathic macular telangiectasia. We describe our experience with an adult patient with a macular edema caused by Leber's miliary aneurysm, which had not been resolved by prior retinal laser photocoagulation, who underwent IVB.

Methods: We investigated the ocular characteristics of the patient before and after a single administration of IVB.

Results: The macular edema had partially improved 1 month after IVB, but then it worsened. We performed laser photocoagulation to treat the aneurysms near the fovea. The macular edema improved, but there was no improvement in visual acuity.
\end{abstract}

Conclusion: In this patient, the effect of IVB was limited.

\section{Introduction}

Leber's miliary aneurysm is a variant of Coats' disease and similar to type 1 idiopathic macular telangiectasia (IMT). Gamulescu et al. [1] reported on an adult case of type 1 IMT that had remarkably improved by an intravitreal injection of bevacizumab (IVB). Lin et al. [2] also concluded that IVB was a well-tolerated treatment for children with Coats' disease; in all 3 patients in their study, the subretinal fluid was completely reabsorbed after they received IVB. We hypothesized that IVB would also be effective in the treatment of a macular edema caused by Leber's miliary aneurysm; therefore, we administered this treatment to an adult patient with this condition. To 
the best of our knowledge, this case is the first report of IVB to treat a macular edema caused by Leber's miliary aneurysm.

\section{Case Report}

A 60-year-old healthy male visited our hospital because he was found to have low visual acuity in his left eye at an examination to renew his driver's license. On his first visit, his best corrected visual acuity (BCVA) was 1.0 and 0.5 for his right and left eye, respectively. In his left eye, at 6 to 9 o'clock of the retina, we found aneurysms, widespread exudative lesions, serous retinal detachment, dilated vessels, and retinal hemorrhages. Focal exudative lesions with hard exudates and retinal hemorrhages were found at 1 and 4 o'clock of the mid-peripheral retina. A macular edema, hard exudates, and some aneurysms were found in the posterior pole. There was mild vitreous opacity. Fluorescein angiography revealed many aneurysms with leakage at 6 to 9 o'clock of the retina, at 1 and 4 o'clock of the mid-peripheral retina, and near the macula (fig. 1a). Optical coherence tomography showed a moderate cystoid macular edema and subretinal fluid ( $\underline{\text { fig. } 2}$ a). There were no abnormal findings in the cornea, anterior chamber, and lens. No particular lesions were found in the right eye. Based on these findings, we diagnosed the patient with Leber's miliary aneurysm. We performed laser photocoagulation of the aneurysms, except near the fovea. Although we assumed that the macular edema was caused by the aneurysms near the fovea, we did not perform laser photocoagulation so as not to injure the macula by this initial treatment. As there was no change in the macular edema or BCVA, IVB (1.25 mg of bevacizumab) was administered 1 month after laser photocoagulation, after informed consent was obtained from the patient. The retinal thickness in the macular region was comparatively decreased 1 month after IVB (fig. 2b), but his BCVA remained 0.6. A fundus photograph 1 month after IVB is shown in figure 1b. Serous retinal detachment found at 6 to 9 o'clock of the peripheral retina had improved. Two months after IVB, the macular edema began to worsen again. Six months after IVB, the macular edema became more severe than it had been before IVB (fig. 2c), and the BCVA decreased to 0.4 , accordingly. The patient did not want to undergo a second administration of IVB, because he felt that there had not been sufficient improvement after the first administration of IVB. To treat the continuing macular edema, we performed laser photocoagulation of the aneurysms near the fovea. Six months after the laser photocoagulation, the macular edema improved (fig. 2d), although the patient's left BCVA remained 0.5.

\section{Discussion}

Treatment with IVB for type 1 IMT, the pathological mechanism of which is similar to Leber's miliary aneurysm, has been reported; but to the best of our knowledge, treatment with IVB for Leber's miliary aneurysm has not been described previously. Gamulescu et al. [1] reported a case of type 1 IMT in whom a single administration of IVB was remarkably effective. In contrast, Takayama et al. [3] and Matsumoto and Yuzawa [4] reported that IVB had limited efficacy for type 1 IMT; the courses of their studies resemble our case. These were all small case series, and further investigations are necessary to determine the efficacy of IVB for this type of aneurysm. In our patient, the effect of IVB on Leber's miliary aneurysm was limited.

Although there was no improvement in the patient's BCVA following the treatment with IVB, there are some points that merit discussion. First, the precise duration of the disease, especially the onset of the macular edema, was unclear; the duration of the edema is sometimes critical for a successful treatment with IVB. Our patient's retinal thickness transiently decreased after IVB, but his BCVA did not improve. It is possible that treatment with IVB might have increased the BCVA in a patient in whom the disease onset was more recent. Second, IVB was administered only once in this case, but repeated IVB might have improved the BCVA, as we have often experienced in 
patients with macular lesions due to age-related macular degeneration and retinal vein occlusion.

The pathological mechanism of Leber's miliary aneurysm is similar to Coats' disease and type 1 IMT. Laser photocoagulation that is generally used as a treatment for Coats' disease and type 1 IMT appeared also effective for Leber's miliary aneurysm in our case.

\section{Disclosure Statement}

The authors have no proprietary interests nor grants and funds received in support of this study to declare.
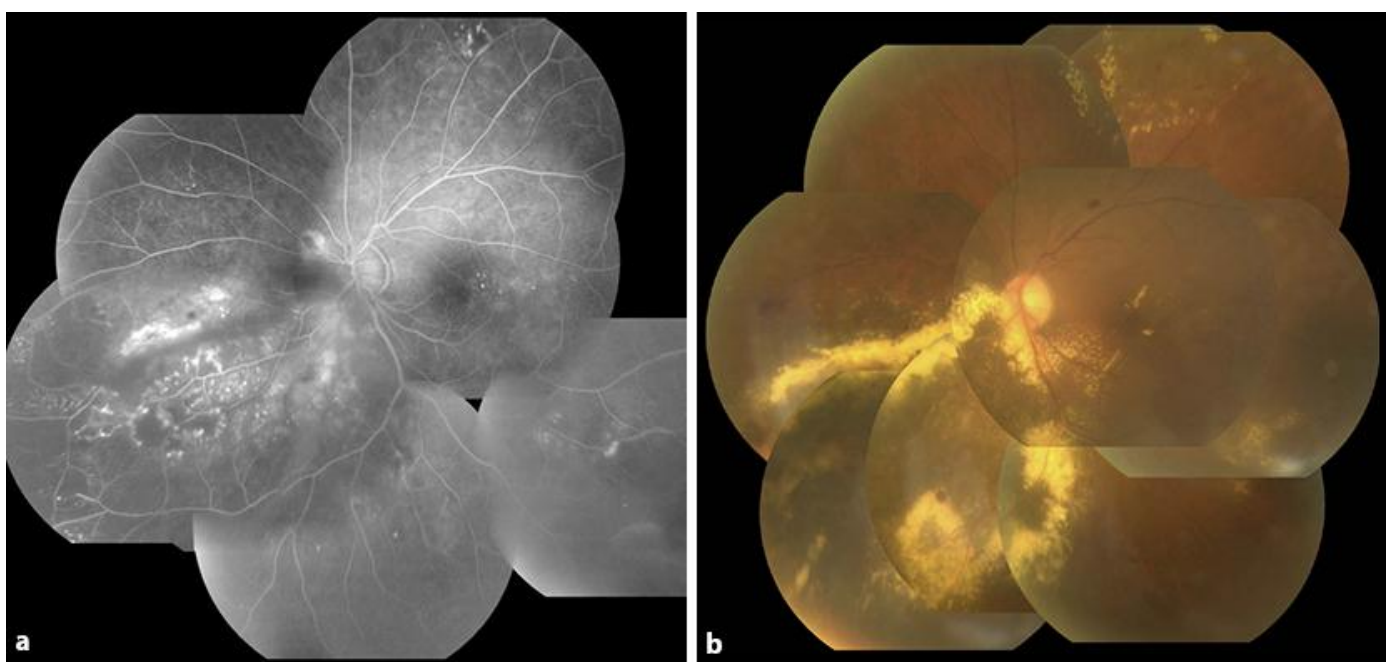

Fig. 1. a Fluorescein angiogram showing aneurysms with leakage at 6 to 9 o'clock of the retina and aneurysms at 1 and 4 o'clock of the mid-peripheral retina and in the parafovea. $\mathbf{b}$ Fundus photograph 1 month after IVB. Laser photocoagulation had been previously performed for the aneurysms except in the parafovea, but massive hard exudates persisted. 

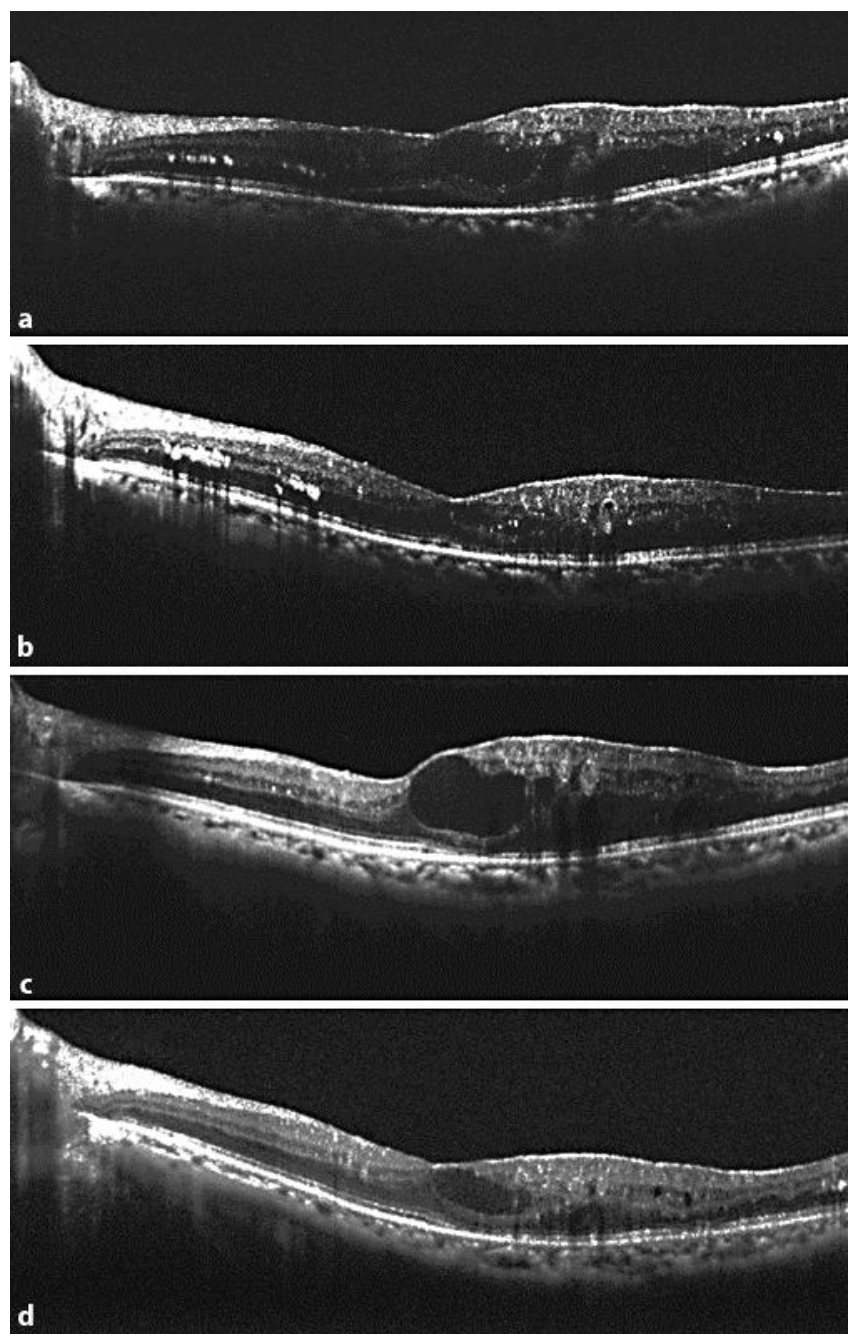

Fig. 2. Macular edema analyzed by optical coherence tomography. a Before IVB. b One month after IVB, the macular edema was comparatively decreased. c Six months after IVB, the macular edema was worse than before IVB. d Six months after laser photocoagulation of the parafoveal aneurysms, the macular edema had improved, but there was no improvement in the BCVA.

\section{References}

1 Gamulescu MA, Walter A, Sachs H, Helbig H: Bevacizumab in the treatment of idiopathic macular telangiectasia. Graefes Arch Clin Exp Ophthalmol 2008;246:1189-1193.

-2 Lin CJ, Hwang JF, Chen YT, Chen SN: The effect of intravitreal bevacizumab in the treatment of Coats disease in children. Retina 2010;30:617-622.

3 Takayama K, Ooto S, Tamura H, Yamashiro K, Otani A, Tsujikawa A, Yoshimura N: Intravitreal bevacizumab for type 1 idiopathic macular telangiectasia. Eye (Lond) 2010;24:1492-1497.

-4 Matsumoto Y, Yuzawa M: Intravitreal bevacizumab therapy for idiopathic macular telangiectasia. Jpn J Ophthalmol 2010;54:320-324. 